\title{
Benchmarks and models for entity-oriented polarity detection
}

\author{
Lidia Pivovarova, Arto Klami, Roman Yangarber \\ University of Helsinki, Finland \\ first.lastecs.helsinki.fi
}

\begin{abstract}
We address the problem of determining entityoriented polarity in business news. This can be viewed as classifying the polarity of the sentiment expressed toward a given mention of a company in a news article. We present a complete, end-to-end approach to the problem. We introduce a new dataset of over 17,000 manually labeled documents, which is substantially larger than any currently available resources. We propose a benchmark solution based on convolutional neural networks for classifying entity-oriented polarity. Although our dataset is much larger than those currently available, it is small on the scale of datasets commonly used for training robust neural network models. To compensate for this, we use transfer learning - pre-train the model on a much larger dataset, annotated for a related but different classification task, in order to learn a good representation for business text, and then fine-tune it on the smaller polarity dataset.
\end{abstract}

\section{Introduction}

We report on research done in the context of PULS-a project for monitoring business news media (Du et al., 2016; Huttunen et al., 2013). ${ }^{1}$ The system gathers 8,000-10,000 documents daily; each document is processed by a cascade of classifiers, including a named entity (NE) recognizer. A key NE in business news type is company or organization, which can be mentioned in a positive or negative context. For example, launching a new product or signing a new contract is viewed as a positive event; involvement in a product recall, bankruptcy or fraud is considered negative.

We focus on determining the polarity of a mention of a given company in news media. Polarity classification is important, since if a company appears in negative contexts frequently, it may affect

\footnotetext{
${ }^{1}$ http://newsweb. cs.helsinki.fi or http://puls.cs.helsinki.fi
}

its reputation, impact its stock price, etc. Polarity prediction, as defined here, is similar to sentiment analysis (Liu and Zhang, 2012): both require the system to classify a span of text as positive or negative. However, there are crucial differences. Business news articles typically do not aim to express emotion or subjectivity-positive and negative events are usually described in a neutral tone. Thus, vocabularies of affective terms-e.g., amazing or terrific - commonly used in sentiment analysis, are not helpful for business polarity. Analysis should rather focus on affective events (Ding and Riloff, 2016), i.e., stereotypically positive or negative events. Further, business news employs genre-specific word usage; words seen as negative in "generic" contexts, may indicate a positive context here, and vice versa.Negative terms in (Hu and Liu, 2004), e.g., include "cancer", which in business often appears in positive contexts, as when a pharmaceuticals unveil novel treatments.

While most work in sentiment analysis is done at the document level, we aim to classify entity mentions in text. This requires changes to documentbased classification models. We explore two convolutional neural network $(\mathrm{CNN})$ architectures, initially proposed for document-level classification, and adapt them for entity-oriented classification. The modified models have an additional input channel: the focus - the position(s) in text where a target company is mentioned. Focus helps the model distinguish among different companies mentioned in text and assign them polarity independently.

As far as we aware no suitable datasets exist for training models for entity-oriented polarity classification. We annotated a dataset of over 17,000 business news articles, which we release for public use, to provide a foundation for an eventual standard evaluation. Despite being much larger than any existing datasets for business polarity detection, it is still small compared to what is typically 
used when training CNNs for text classification.

We attempt to compensate for the small training data by transferring knowledge from a different corpus. The second corpus is large, but annotated for a different task: each document has a set of event labels; some of these may be mapped to polarity labels. We explore two strategies for knowledge transfer: i) manually mapping from event labels to polarity labels, and ii) pre-training CNNs for the event classification task, followed by unsupervised transfer of high-level features from event classification to polarity. We demonstrate that unsupervised transfer improves performance.

\section{Related work}

Sentiment analysis: Deep learning for sentiment analysis is an active area of research. Some methods learn vector representations for entire phrases (Dos Santos and Gatti, 2014; Socher et al., 2011); others learn syntactic tree structures (Tai et al., 2015; Socher et al., 2013). A simpler approach using CNNs (Kim, 2014) has demonstrated state-of-the-art performance (Tai et al., 2015).

Interest in applying sentiment mining to the business domain is spurred by important industry applications, such as analyzing the impact of news on financial markets (Ahmad et al., 2016; Van de Kauter et al., 2015; Loughran and McDonald, 2011). If a company frequently appears in news in negative contexts it may affect its reputation, impact its stock price, etc., (Saggion and Funk, 2009). Although news reports usually have a time lag, events reported in news have longer-term impact on investor sentiment and attitudes toward a given company (Boudoukh et al., 2013).

A major difficulty in training entity-oriented polarity models is the lack of publicly available datasets. In the corpus of 5,000 sentences published by Takala et al. (2014), most instances (sentences) contain no company name, and hence cannot be used for predicting polarity for specific entities. A dataset of 679 sentences in Dutch, annotated with entity-oriented business sentiment, was published by Van de Kauter et al. (2015). They demonstrate that a. in financial news, not all sentiment expressions within a sentence relate to the target company; b. sentiment is often expressed implicitly.

A shared task on fine-grained sentiment analysis of financial microblogs and news was held recently as part of SemEval (Cortis et al., 2017), and provided a small dataset containing company names.
This dataset contains only 1,000 news headlines, of which only 165 instances mention more than one company name, of which only 20 instances contain names with different polarities (positive for one company but negative for another). Thus, using entity-oriented methods on this dataset may not lead to an advantage in performance. Of the ten best-performing systems on the news sentiment task, many used sentence-level classification with no treatment of target company (Rotim et al., 2017; Cabanski et al., 2017; Ghosal et al., 2017; Kumar et al., 2017); others replace the target name with a special token (Mansar et al., 2017; Moore and Rayson, 2017; Jiang et al., 2017) or use company name as a feature (Kar et al., 2017), though none of the papers provide any evidence that special treatment of the target yields a gain in performance. In our experiments with the SemEval dataset (Pivovarova et al., 2017) a model with explicitly specified target worked slightly worse than a baseline.

The dataset that we release with this paper is 20 times larger and contains entire documents, where a given entity may be mentioned multiple times, with many different names mentioned in the same document. This corpus is suitable for experiments with entity-oriented polarity, and our experiments explicitly contrast models that take focus as an input against models that do not use the information about the target company's position.

Transfer learning: a.k.a. inductive transfer, is a technique for applying knowledge accumulated from solving one problem to improve the solution for a different problem. We use feature transfer, where the goal is to learn transferable representations for data, which are meaningful for multiple tasks (Pan and Yang, 2010; Bengio et al., 2013; Conneau et al., 2017), i.e., very general, low-level representations. On the other hand, one might consider two related tasks, and try to gain knowledge from one to help with the other. In such cases, one wishes to transfer representations at a much higher level (Glorot et al., 2011). An analysis of the trade-offs between generality and specificity of learned features can be found at (Yosinski et al., 2014). Deep learning with knowledge transfer has been previously applied to sentiment analysis in the context of domain adaptation (Glorot et al., 2011) and cross-lingual applications (Zhou et al., 2016). In our experiments, we apply knowledge transfer from event classification to sentiment analysis. 


\section{The Model}

We train a classifier for entity-oriented polarity, which receives on input a text and a "focus" vectorthe positions of mentions of the target company in text-and outputs the polarity for this company. For this purpose we extend state-of-the-art models in (Kim, 2014). The rationale for introducing focus is that polarity is not a feature of the text as a whole, but of each company mention; two company mentions in a text may have opposing polarities, and the model needs be able to distinguish them.

The architecture of the model is shown in Figure 1 . The inputs are fed into the network as sentences of a fixed size, zero-padded; each word is a fixed-dimensional embedding vector complemented with a scalar indicating the focus. The focus vector is shown in darker grey in Figure 1, with the the company mention framed in red. This provides an additional dimension to the word embedding, and is crucial for distinguishing between instances that differ only in focus and polarity.

The inputs are fed into a layer of convolutional filters with multiple widths, optionally followed by deeper convolutional layers. The results of the last convolutional layer are max-pooled, producing a vector with one scalar per filter, which is then fed into a fully-connected layer with dropout regularization and a soft-max output layer. The output is a 2-dimensional vector that is a probability distribution over the two possible outcomes: positive and negative. In manual annotation we use five values: "very negative" [1 0], "somewhat negative" [.7 .3], "neutral" [.5 .5], "somewhat positive" [.3 .7] and "very positive" [0 1]. The model may output any possible distribution. The loss is cross-entropy between the network's output and the true distribution; the loss updates the weights via back-propagation.

We represent words by embeddings, trained using the GloVe algorithm (Pennington et al., 2014) on a corpus of 5 million news articles. Each article was pre-processed using lemmatization and the PULS NE recognition system. All NEs of the same type are mapped to the same special token; i.e., all company names have the same embedding, all person names another, etc. We continue to train the embeddings during polarity training by updating them at each iteration. This allows the model to learn properties of words significant for polarity, such as the difference between antonyms, which may not be captured well by the initial embeddings.

\begin{tabular}{lr|}
\hline Class & \# instances \\
\hline very positive & 2709 \\
positive & 4001 \\
neutral & 285 \\
\hline
\end{tabular}

\begin{tabular}{|lr}
\hline Class & \# instances \\
\hline very negative & 2532 \\
negative & 4645 \\
contradictory & 146 \\
\hline
\end{tabular}

Table 1: Class distribution in annotated data.

\section{Data}

The dataset contains 17,354 different documents with 19,689 company names. PULS clusters news into groups, each group containing documents describing the same story. ${ }^{2}$ Then we manually annotate each group with business polarity of the most salient company names.

In our experiments, each training instance is the first five sentences in the document beginning from the first mention of the focus company. This choice was made because typically the beginning of an article carries information about the principal event, whereas later text contains background information which may mention the company, but where the polarity may be different. In case this processing results in identical instances, we remove duplicates, and keep only one copy.

The resulting dataset used in the experiments contains 14,172 distinct instances. The distribution of the data among the polarity classes is shown in Table 1. Instances labeled "contradictory" are not used for testing and training at present. The data were split into five folds for cross-validation.

We also have a separate, large collection of news articles (Pivovarova et al., 2013), which is annotated for business events-for example, Merger, Contract, Investment, Product launch, Product recall, Fraud, Bankruptcy-291 labels in all. An article may have multiple event labels. Some of these labels may imply (or strongly correlate with) positive or negative polarity. We attempt to exploit this large data to improve polarity prediction. To this end, we attempt two approaches, with several variations: manual mapping and high-level feature transfer.

For manual mapping, we manually selected those labels which we believe most clearly imply a polarity: e.g., Investment, Product launch and Sponsorship are considered positive, while Fraud, Layoff and Bankruptcy are negative; in all, we identified 26 "positive" and 12 "negative" labels. Using

\footnotetext{
${ }^{2}$ The grouping algorithm takes into account the semantic similarity of the keywords, and the distributions of NEs within the documents (Escoter et al., 2017)
} 


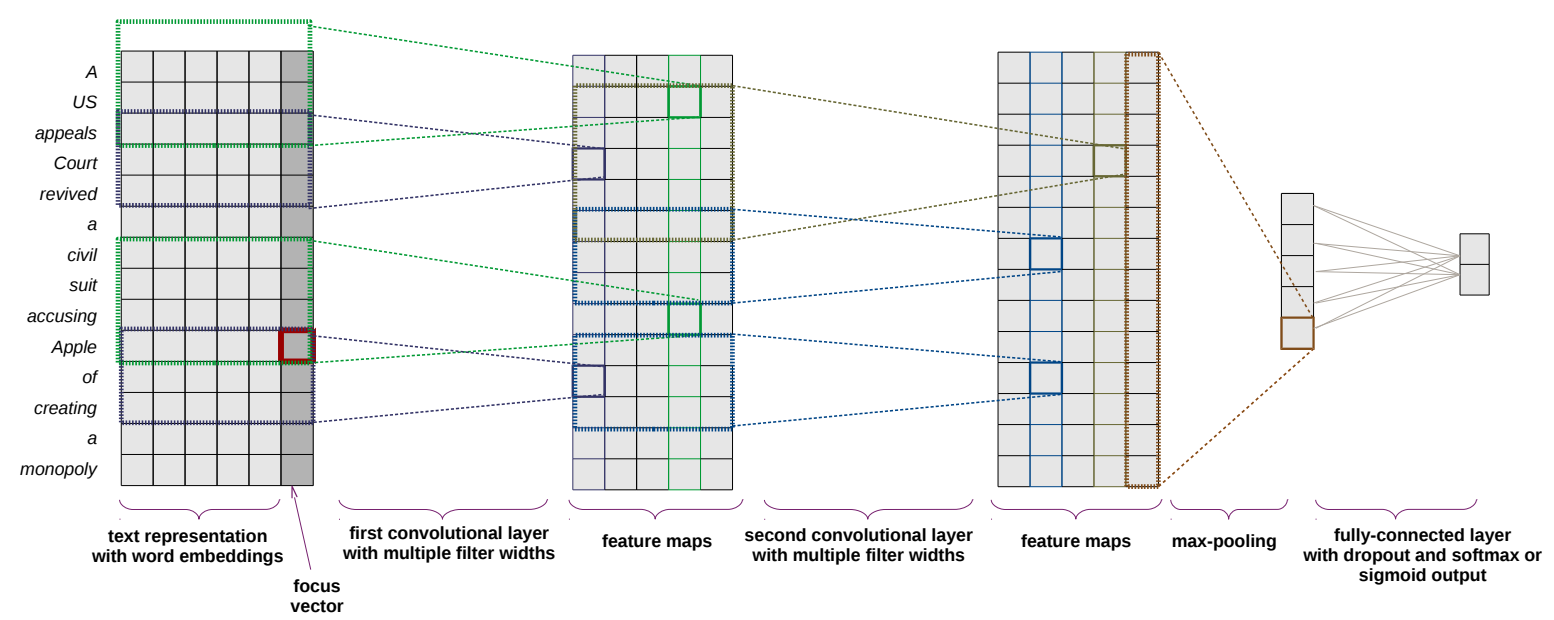

Figure 1: A model architecture with focus vector and two convolution layers

only these 38 event labels, we constructed a training set, removing documents with labels that result in no polarity, or conflicting polarities. Further, since it is impossible to know to which company the label refers, only documents whose headlines and the first sentence contain exactly one company mention were kept. (For example, if one company goes bankrupt and another acquires its assets—-such documents are not used.)

The resulting dataset is highly skewed with $90 \%$ of the data positive. To assure that the positive and negative subsets have similar size, we apply random undersampling (Dendamrongvit and Kubat, 2010), i.e., we use a random subset of the positive documents. Of more than two million documents in the original event corpus, 100,000 have a nonambiguous negative label and mention exactly one company. The resulting dataset consists of 200,000 documents; $10 \%$ is used as a development set to decide when to stop training. We use this newly generated $200 \mathrm{~K}$ document event corpus in 2 ways:

Tuning: a two-stage learning procedure where the model is first trained using the event corpus, and then is tuned using the smaller polarity corpus.

Training on combined data: in this strategy, data from both corpora are mixed together, and used for training in random order.

In high-level feature transfer, we aim to reuse relatively high-level, task-specific features. We initially train a model to predict event types-using all event labels and all documents, irrespective of how many companies they mention. This requires a change in the models: because event labels are not mutually exclusive, we use a sigmoid function instead of soft-max in the topmost layer. After the event model is fully trained on the event labels, we strip off the last fully-connected layer of the network, replace it with a two-class output layer for polarity, and resume training using the smaller polarity dataset. We expect that the more task-specific features - ones obtained closer to the output layerwill be useful to determine polarity values, due to the latent relatedness between the two tasks. Thus, we keep almost the entire model, with the exception of the very final layer.

From the large dataset labeled with events, we use $10 \%$ as a development set, to determine when to stop training, and to find the best model; another $10 \%$ is used as a test set. Results from representative runs are shown in Table 2.

The bigger model gives better performancewith a much larger number of filters (1000 vs. 128). It is not possible to use such large models for learning polarity without transfer, because these models are trained on much smaller data and would quickly overfit. Therefore, in subsequent experiments we use two convolutional layers with filter sizes $3,4,5$, with 128 filters of each size. ${ }^{3}$

\section{Experiments}

We present experiments with focus and knowledge transfer variants. Table 3 shows the results for each model variant, averaged across five-fold crossvalidation. We report accuracy and cosine similar-

\footnotetext{
${ }^{3}$ The overall accuracy of the method can likely be improved further by careful tuning of hyper-parameters. Here we focus on the comparison of architectures, and defer hyperparameters for future work.
} 


\begin{tabular}{l|c|c|c|}
\hline \multicolumn{1}{c|}{ Hyper-Parameters } & rec & prec & F1 \\
\hline conv 2; filters 128; sizes $\{3,4,5\}$ & 35.85 & 75.42 & 48.53 \\
conv 1; filters 1000; sizes $\{3,7,11\}$ & 50.80 & 58.13 & $\mathbf{5 3 . 8 9}$ \\
\hline
\end{tabular}

Table 2: Event classification results

\begin{tabular}{l|l|r|r}
\hline Transfer Strategy & Focus & Accuracy & Cosine \\
\hline none & - & 81.22 & 71.51 \\
none & + & 81.44 & 72.93 \\
manual tuning & + & 82.07 & $\mathbf{7 3 . 9 8}$ \\
manual combined data & + & 82.01 & 70.92 \\
\hline feature transfer & - & 83.94 & 71.17 \\
feature transfer & + & $\mathbf{8 4 . 4 4}$ & 71.76 \\
\hline baseline (SVM) & & 52.02 &
\end{tabular}

Table 3: Experimental results (multiplied by 100, for readability.)

ity between the model output and the annotation.

To compute accuracy as follows. In annotation we treat polarity detection as a three-way classification task; values inside $[-0.1,0.1]$ are considered neutral; values further from 0.0 are positive or negative. However, for reasons presented below, the models do not do well on identifying neutral instances. Thus, in the experiments presented here, we evaluate prediction of binary polarity ${ }^{4}$ : negative vs. positive or neutral. Accuracy measures how often a model blunders, and predicts negative polarity rather than positive or neutral, or vice versa.

Cosine similarity ${ }^{5}$ is computed by collecting all of the model's polarity probabilities into one vector and one for the manually assigned polarities, and measuring the cosine between the vectors; also, polarities are mapped into the interval $[-1,1]$. This gives a measure of closeness between model prediction and the ground truth, including differences between "positive" and "very positive" classes.

As the results show, accuracy and cosine similarity do not produce consistent rankings, because they measure different aspects of performance. From a practical, user-oriented point of view, it may be more important that a model avoid gross errors, rather than capturing subtle shades of polarity. In manual annotation we noticed that some distinctions ("positive" vs. "very positive") is far from

\footnotetext{
${ }^{4}$ Due to the industry-level requirements: in business news negative polarity has important implications; "neutral" coverage may even be viewed mildly positive, as the entity mentioned is receiving (non-negative) publicity, etc.

${ }^{5}$ This is the official measure of the SemEval business polarity classification task (Cortis et al., 2017); we include it because it may be useful for indirect comparison of results.
}

clear for human annotators. Thus, we are interested in the models that yield the best accuracy.

In addition, we used a SVM classifier as a baseline. The baseline does not use any information about the target company. We use a one-vs-all strategy to obtain three-way classification. For the baseline we report only the accuracy, since this method does not directly produce probabilities.

\section{Discussion}

Knowledge transfer: Table 3 shows that highlevel feature transfer outperforms manual mapping. The main reason might be that feature transfer can benefit from a very large corpus of 2 million documents, while only 200,000 documents can be used with the manual mapping approach, which prevents us from training larger models due to over-fitting. The mapped dataset may suffer from other problems, resulting from how it is created. First, it contains no articles with neutral polarity-if an article has no positive or negative label, we cannot assume it to be neutral. For example, articles labeled Corporate appointments may have positive or negative polarity. Second, although we choose only the most "trusted" event labels for mapping to polarity, the dataset still contains noise: e.g., a document labeled as Merger and assumed to be positive may in fact discuss a canceled merger. Third, since we use only a small subset of the labels, the dataset is highly skewed and incomplete-most event types and data are not used. Most importantly, using manually mapped data, a model is trained to perform a task different from our target-it learns to dis- 


\begin{tabular}{|c|l|r|r|r|l|}
\hline 1 & Example & Truth & +Focus & -Focus & Comment \\
\hline Valeant to sell Dendreon unit to Sanpower for \$820 \\
million. Canada's Valeant Pharmaceuticals Inter- \\
national Inc. said its affiliate will sell its Dendreon \\
cancer business to Sanpower Group Co. Ltd. for \\
$\begin{array}{l}\$ 819.9 \text { million, as the drugmaker continues to shed } \\
\text { its non-core assets to repay debt. }\end{array}$ & -1.0 & 0.022 & -0.322 & $\begin{array}{l}\text { The model without focus per- } \\
\text { forms better since the company } \\
\text { name is mentioned far away } \\
\text { from the polarity expression and } \\
\text { there is another name in be- } \\
\text { tween. }\end{array}$ \\
\hline 2 & $\begin{array}{l}\text { Samsung wins over Apple in \$399 million patent } \\
\text { appeal. }\end{array}$ & -1.0 & -0.333 & 0.004 & $\begin{array}{l}\text { Model without focus fails: two } \\
\text { companies with opposite polari- } \\
\text { ties involved in same event. }\end{array}$ \\
\hline 3 & $\begin{array}{l}\text { Facebook CEO Mark Zuckerberg and his wife are } \\
\text { dropping controversial suits they filed in December } \\
\text { to buy small plots of land that are part of a [...] } \\
\text { estate they own on the island of Kauai in Hawaii. }\end{array}$ & 0.0 & -0.743 & -0.397 & $\begin{array}{l}\text { Text is about Facebook CEO, } \\
\text { not the company itself. None of } \\
\text { the models handle neutral com- } \\
\text { pany mentions well. }\end{array}$ \\
\hline
\end{tabular}

Table 4: Model comparison: CNN with/without focus using transfer. Company in focus is in bold

tinguish not positive vs. negative polarity, but one (sub-)set of event labels from another. We cannot assume that the model learns polarity patterns, only that polarity correlates with certain event types.

Focus: The results indicate that focus further improves performance. ${ }^{6}$ On some test instances, models without focus outperforms models with focus - this happens when polarity expressions lie outside the filter window around the focus company, e.g., as in Example 1 in Table 4.

If two companies within the same text have opposite polarities, a model without focus can assign the correct polarity to at most one of them, as in example 2 in Table 4. Such cases are rare in our dataset; typically, when two companies are involved in the same event, they have the same polarity, e.g., when they strike a deal. Only $6 \%$ of instances in our dataset have a paired instance that has identical text but different focus and opposite polarity. Another case when focus is useful is when a document contains much background information, which may contain opposite polarity statements. Estimating the number of such cases is an arduous task. Since in some cases a model with focus performs worse than a model without focus, there is no clear gain in that regard. However, the best-performing transfer strategy works slightly better, as seen from Table 3 .

Neutral polarity: Another observation is that all of our models have difficulty in detecting neutral polarities, as shown in Example 3 (which is about Facebook's CEO, rather than the company itself). Neutral examples are rare in our dataset, as shown in Table 1 . This is probably the main reason why the models are unable to distinguish neutral polarity. This problem may be helped by annotating more neutral instances.

\footnotetext{
${ }^{6}$ Results are statistically significant at $p<0.05$ or lower.
}

\section{Conclusion}

We address the problem of entity-oriented business polarity detection. The main contributions are: I. a dataset of 17,000 annotated documents, which is an order of magnitude larger than any previously available resources for this task $;{ }^{7}$ II. we propose benchmark solutions to this problem, based on CNN architectures originally intended for document-level polarity classification, modified for entity-oriented polarity classification by explicitly incorporating focus into the model; III. we demonstrate that performance can be improved via transfer learning, by training a network on a much larger corpus, which is annotated for a different, distantly related task-namely, classification of event types.

We compare manual label mapping with transferring high-level features, and demonstrate that the latter approach performs better, and is less subjective; i.e., features relevant for finding event types work better than a simplistic mapping between the two tasks. The rationale behind this is that business polarity is latently inherent in the event types themselves: some event types carry a positive or negative polarity, while others do not indicate an unambiguous polarity. Therefore, attempting to map event labels directly to polarity is problematic.

For manual mapping of event labels, we can use only documents with exactly one company and "unambiguous" event labels, while for transfer learning we can use the entire event dataset, which lets us use much more data for training bigger models.

High-level feature transfer yields $15.8 \%$ error reduction $-81 \%$ to $84 \%$ accuracy-as compared to using only the small polarity-annotated corpus.

\footnotetext{
${ }^{7}$ The corpus is available at puls.cs.helsinki.fi/ polarity
} 


\section{Acknowledgements}

This work was supported in part by Tekes (the Finnish Funding Agency for Technology and Innovation) Project iMEDL: Digital Media Evolution through Deep Learning, Grant number 5933/31/2017.

\section{References}

Khurshid Ahmad, JingGuang Han, Elaine Hutson, Colm Kearney, and Sha Liu. 2016. Media-expressed negative tone and firm-level stock returns. Journal of Corporate Finance 37:152-172.

Yoshua Bengio, Aaron Courville, and Pascal Vincent. 2013. Representation learning: A review and new perspectives. IEEE transactions on pattern analysis and machine intelligence 35(8):1798-1828.

Jacob Boudoukh, Ronen Feldman, Shimon Kogan, and Matthew Richardson. 2013. Which news moves stock prices? A textual analysis. Technical report, National Bureau of Economic Research.

Tobias Cabanski, Julia Romberg, and Stefan Conrad. 2017. HHU at SemEval-2017 task 5: Finegrained sentiment analysis on financial data using machine learning methods. In Proceedings of the 11th International Workshop on Semantic Evaluation (SemEval-2017). pages 832-836.

Alexis Conneau, Douwe Kiela, Holger Schwenk, Loic Barrault, and Antoine Bordes. 2017. Supervised learning of universal sentence representations from natural language inference data

Keith Cortis, André Freitas, Tobias Dauert, Manuela Huerlimann, Manel Zarrouk, Siegfried Handschuh, and Brian Davis. 2017. Semeval-2017 task 5: Finegrained sentiment analysis on financial microblogs and news. In Proceedings of the 11th International Workshop on Semantic Evaluation (SemEval-2017). Association for Computational Linguistics, Vancouver, Canada, pages 510-526.

Sareewan Dendamrongvit and Miroslav Kubat. 2010. Undersampling approach for imbalanced training sets and induction from multi-label textcategorization domains. In New Frontiers in Applied Data Mining, Springer, pages 40-52.

Haibo Ding and Ellen Riloff. 2016. Acquiring knowledge of affective events from blogs using label propagation. In Thirtieth AAAI Conference on Artificial Intelligence.

Cícero Nogueira Dos Santos and Maira Gatti. 2014. Deep convolutional neural networks for sentiment analysis of short texts. In COLING. pages 69-78.
Mian Du, Lidia Pivovarova, and Roman Yangarber. 2016. PULS: natural language processing for business intelligence. In Proceedings of the 2016 Workshop on Human Language Technology. Go to Print Publisher, pages 1-8.

Llorenç Escoter, Lidia Pivovarova, Mian Du, Anisia Katinskaia, and Roman Yangarber. 2017. Grouping business news stories based on salience of named entities. In 15th Conference of the European Chapter of the Association for Computational Linguistics Proceedings of Conference, Volume 1: Long Papers.

Deepanway Ghosal, Shobhit Bhatnagar, Md Shad Akhtar, Asif Ekbal, and Pushpak Bhattacharyya. 2017. IITP at SemEval-2017 task 5: an ensemble of deep learning and feature based models for financial sentiment analysis. In Proceedings of the 11th International Workshop on Semantic Evaluation (SemEval-2017). pages 899-903.

Xavier Glorot, Antoine Bordes, and Yoshua Bengio. 2011. Domain adaptation for large-scale sentiment classification: A deep learning approach. In Proceedings of the 28th international conference on machine learning (ICML-11). pages 513-520.

Minqing $\mathrm{Hu}$ and Bing Liu. 2004. Mining and summarizing customer reviews. In Proceedings of the tenth ACM SIGKDD international conference on Knowledge discovery and data mining.

Silja Huttunen, Arto Vihavainen, Mian Du, and Roman Yangarber. 2013. Predicting relevance of event extraction for the end user. In T. Poibeau, H. Saggion, J. Piskorski, and R. Yangarber, editors, Multi-source, Multilingual Information Extraction and Summarization, Springer Berlin, Theory and Applications of Natural Language Processing, pages 163-176.

Mengxiao Jiang, Man Lan, and Yuanbin Wu. 2017. ECNU at SemEval-2017 task 5: An ensemble of regression algorithms with effective features for finegrained sentiment analysis in financial domain. In Proceedings of the 11th International Workshop on Semantic Evaluation (SemEval-2017). pages 888893.

Sudipta Kar, Suraj Maharjan, and Thamar Solorio. 2017. RiTUAL-UH at SemEval-2017 task 5: Sentiment analysis on financial data using neural networks. In Proceedings of the 11th International Workshop on Semantic Evaluation (SemEval-2017). pages $877-882$.

Yoon Kim. 2014. Convolutional neural networks for sentence classification. In Proceedings of the Conference on Empirical Methods in Natural Language Processing (EMNLP).

Abhishek Kumar, Abhishek Sethi, Md Shad Akhtar, Asif Ekbal, Chris Biemann, and Pushpak Bhattacharyya. 2017. IITPB at SemEval-2017 task 5: Sentiment prediction in financial text. In Proceedings of the 11th International Workshop on Semantic Evaluation (SemEval-2017). pages 894-898. 
Bing Liu and Lei Zhang. 2012. A survey of opinion mining and sentiment analysis. In Mining text data, Springer, pages 415-463.

Tim Loughran and Bill McDonald. 2011. When is a liability not a liability? textual analysis, dictionaries, and 10-ks. The Journal of Finance 66(1):35-65.

Youness Mansar, Lorenzo Gatti, Sira Ferradans, Marco Guerini, and Jacopo Staiano. 2017. Fortia-FBK at SemEval-2017 task 5: Bullish or bearish? inferring sentiment towards brands from financial news headlines. arXiv preprint arXiv:1704.00939.

Andrew Moore and Paul Rayson. 2017. Lancaster A at SemEval-2017 task 5: Evaluation metrics matter: predicting sentiment from financial news headlines. arXiv preprint arXiv:1705.00571.

Sinno Jialin Pan and Qiang Yang. 2010. A survey on transfer learning. IEEE Transactions on knowledge and data engineering 22(10):1345-1359.

Jeffrey Pennington, Richard Socher, and Christopher D. Manning. 2014. GloVe: Global vectors for word representation. In Empirical Methods in Natural Language Processing (EMNLP). pages 1532-1543.

Lidia Pivovarova, Llorenç Escoter, Arto Klami, and Roman Yangarber. 2017. HCS at SemEval-2017 task 5: Polarity detection in business news using convolutional neural networks. In Proceedings of the 11th International Workshop on Semantic Evaluation (SemEval-2017). pages 842-846.

Lidia Pivovarova, Silja Huttunen, and Roman Yangarber. 2013. Event representation across genre. In Proceedins of the $1^{\text {st }}$ Workshop on Events: Definition, Detection, Coreference, and Representation. NAACL HLT.

Leon Rotim, Martin Tutek, and Jan Šnajder. 2017. TakeLab at SemEval-2017 task 5: Linear aggregation of word embeddings for fine-grained sentiment analysis of financial news. In Proceedings of the 11th International Workshop on Semantic Evaluation (SemEval-2017). pages 866-871.

Horacio Saggion and Adam Funk. 2009. Extracting opinions and facts for business intelligence. RNTI Journal, E (17) 119:146.

Richard Socher, Jeffrey Pennington, Eric H. Huang, Andrew Y. Ng, and Christopher D. Manning. 2011. Semi-supervised recursive autoencoders for predicting sentiment distributions. Association for Computational Linguistics, Stroudsburg, PA, USA, EMNLP'11.

Richard Socher, Alex Perelygin, Jean Y Wu, Jason Chuang, Christopher D Manning, Andrew Y Ng, and Christopher Potts. 2013. Recursive deep models for semantic compositionality over a sentiment treebank. In Proceedings of the conference on empirical methods in natural language processing (EMNLP). Citeseer, volume 1631, page 1642.
Kai Sheng Tai, Richard Socher, and Christopher D Manning. 2015. Improved semantic representations from tree-structured long short-term memory networks. In $A C L$.

Pyry Takala, Pekka Malo, Ankur Sinha, and Oskar Ahlgren. 2014. Gold-standard for topic-specific sentiment analysis of economic texts. In $L R E C$. pages 2152-2157.

Marjan Van de Kauter, Diane Breesch, and Véronique Hoste. 2015. Fine-grained analysis of explicit and implicit sentiment in financial news articles. Expert Systems with Applications .

Jason Yosinski, Jeff Clune, Yoshua Bengio, and Hod Lipson. 2014. How transferable are features in deep neural networks? In Advances in neural information processing systems. pages 3320-3328.

Guangyou Zhou, Zhao Zeng, Jimmy Xiangji Huang, and Tingting He. 2016. Transfer learning for crosslingual sentiment classification with weakly shared deep neural networks. In Proceedings of the 39th International ACM SIGIR conference on Research and Development in Information Retrieval. 